Noname manuscript No.

(will be inserted by the editor)

O. A. Rodríguez · M. A. Solís

\title{
Universal behavior of the BEC critical temperature for a multislab ideal Bose gas
}

Received: date / Accepted: date

\begin{abstract}
For an ideal Bose-gas within a multi-slab periodic structure, we discuss the effect of the spatial distribution of the gas on its Bose-Einstein condensation critical temperature $T_{c}$, as well as on the origin of its dimensional crossover observed in the specific heat. The multi-slabs structure is generated by applying a Kronig-Penney potential to the gas in the perpendicular direction to the slabs of width $b$ and separated by a distance $a$, and allowing the particles to move freely in the other two directions. We found that $T_{c}$ decreases continuously as the potential barrier height increases, becoming inversely proportional to the square root of the barrier height when it is large enough. This behavior is universal as it is independent of the width and spacing of the barriers. The specific heat at constant volume shows a crossover from $3 \mathrm{D}$ to $2 \mathrm{D}$ when the height of the potential or the barrier width increase, in addition to the well known peak related to the Bose-Einstein condensation. These features are due to the trapping of the bosons by the potential barriers, and can be characterized by the energy difference between the energy bands below the potential height.
\end{abstract}

Keywords Bose gas · multi-slabs · critical temperature

\section{Introduction}

Quantum fluids within periodic structures have been subject of study from some decades ago. Important examples of this kind of systems are electron gas in layered cuprate superconductors [1], cold atomic gases in periodic structures constructed from laser beams known as optical lattices [2], the

O. A. Rodríguez López

Posgrado en Ciencias Físicas, UNAM, E-mail: oarodriguez@fisica.unam.mx

M. A. Solís

Instituto de Física, UNAM, E-mail: masolis@fisica.unam.mx 
well known liquid helium four or three in any dimension [3], electons or holes in semiconductor superlattices 4] or Cooper pairs in tube bundle superconductors 5 . The physical properties of a constrained quantum gas come from the effects of the external potentials and the interactions between particles. Here we are giving a detailed description of the effects of the constraints on the Bose gas properties before to address a more complete description including interactions. Let us consider a 3D ideal Bose gas (IBG) within a lattice composed by multiple slabs. The bosons are free to move in the $x$ an $y$ directions. The lattice is modeled through a Kronig-Penney potential in the $z$ direction, with potential barriers of width $b$ separated a distance $a$, so the potential period, or the lattice period, is $a+b$. We solve the Schrödinger equation by separation of variables. The energy of the particles with mass $m$ is $\varepsilon=\varepsilon_{x}+\varepsilon_{y}+\varepsilon_{z}$, where $\varepsilon_{x}=\hbar^{2} k_{x}^{2} / 2 m, \varepsilon_{y}=\hbar^{2} k_{y}^{2} / 2 m$, being $\hbar k_{x}$ and $\hbar k_{y}$ the momentum of free bosons in the $x$ and $y$ directions respectively, while $\varepsilon_{z}$ is found by solving the Schrödinger equation in the $z$ coordinate with the Kronig-Penney potential

$$
\mathcal{U}(z)=V_{0} \sum_{n=-\infty}^{\infty} \Theta[z-(n-1)(a+b)-a] \Theta[n(a+b)-z],
$$

where $\Theta(z)$ is the Heaviside step function and $V_{0}$ is the height of the potential barriers. The allowed energies for a particle in the $z$-direction are given by 6]

$$
\frac{V_{0}-2 \varepsilon_{z}}{2 \sqrt{\varepsilon_{z}\left(V_{0}-\varepsilon_{z}\right)}} \sinh (\kappa b) \sin (\alpha a)+\cosh (\kappa b) \cos (\alpha a)=\cos \left[k_{z}(a+b)\right],
$$

where $\hbar \kappa=\sqrt{2 m\left(V_{0}-\varepsilon_{z}\right)}$ and $\hbar \alpha=\sqrt{2 m \varepsilon_{z}}$.

The energy spectrum consist of a series of allowed energy bands separated by forbidden regions, where the positive side of the $j$-th band extends from $k_{z}(a+b)=(j-1) \pi$ to $j \pi$ with $j=1,2,3, \ldots$. The Fig. 1 in Ref. 8] shows the energy spectrum, and the allowed energy bands, in terms of the dimensionless parameters $u_{0}=\left(2 m(a+b)^{2} / \hbar^{2}\right) V_{0}, \bar{\varepsilon}_{z}=\left(2 m(a+b)^{2} / \hbar^{2}\right) \varepsilon_{z}$ and $r=b / a$, for $r=1$. Pi squared times our energy unit $\hbar^{2} / 2 m(a+b)^{2}$ is equivalent to the recoil energy of an 1D optical lattice formed by the interference of two counter-propagating light beams of wavelength equal to $2(a+b)$. The parameter $u_{0}$ represents the lattice height (or depth) in our energy unit, and the quotient $r$ indicates how wide are the barriers with respect to the lattice period. It also indicates if a lattice is square $(r=1)$ or rectangular $(r \neq 1)$.

\section{Universal behavior of the critical temperature}

We obtain the thermodynamic properties of the Bose gas from the Grand Potential $\Omega=U-T S-\mu N$. In order to find the Bose-Einstein critical temperature $T_{c}$ we use the number equation, $N=N_{0}+N_{e}$, where $N_{0}$ is the number of bosons occupying the ground state, and $N_{e}$ is the number of bosons distributed over excited states. At $T=T_{c}, \mu\left(T_{c}\right)=\mu_{0}=\varepsilon_{0}$ is the 
energy of the ground state of the system, and all the bosons are distributed over the excited states, $N \approx N_{e}$. The number equation becomes

$$
N=-\frac{m V}{(2 \pi)^{2} \hbar^{2}} \frac{1}{\beta_{c}} \int_{-\infty}^{\infty} d k_{z} \ln \left(1-e^{-\beta_{c}\left(\varepsilon_{z}-\mu_{0}\right)}\right),
$$

with $\beta_{c}=1 / k_{B} T_{c}$. From the last Eq. (3) we obtain the critical temperature $T_{c}$ in an implicit way, which is numerically calculated. Our system size is infinite, and it has an infinite number of bosons, but the average particle density $\eta_{b}=N / V$ is a constant. An infinite IBG with the same average density but with no external potential has a critical temperature given by $T_{0}=\left(2 \pi \hbar^{2} / m k_{B}\right)\left[\eta_{b} / \zeta(3 / 2)\right]^{2 / 3}$, where $\zeta(3 / 2) \simeq 2.612$ is the Riemann Zeta function of argument $3 / 2$. It turns out that $T_{0}$ is a good reference parameter for temperatures as well as the thermal wavelength $\lambda_{0}=h / \sqrt{2 \pi m k_{B} T_{0}}$ is a good reference parameter for lengths, in particular for the critical temperature $T_{c}$ and the potential spatial period $a+b$, respectively.

We previously found 8 that, when $u_{0} \equiv\left(2 m(a+b)^{2} / \hbar^{2}\right) V_{0} \gg 1$ the critical temperature is inversely proportional to the square root of this parameter. However, in order to observe separately the effects of the spatial period variation as well as the increasing of the potential height on the critical temperature, in this Section we find it convenient to replace $u_{0}$ by $\tilde{u}_{0} \equiv\left(2 m \lambda_{0}^{2} / \hbar^{2}\right) V_{0}$ which is period independent and it depends exclusively on changes of the potential magnitude $V_{0}$. The behavior of the critical temperature as a function of $\tilde{u}_{0}$ (instead of $u_{0}$ ) is shown in Fig. 1, for $r=1$, i.e., the width of the barriers is equal to the separation between them. In the same figure each curve of $T_{c} / T_{0}$ corresponds to a particular value of the period of the potential. We can see that $T_{c}$ is a monotonically decreasing function of $\tilde{u}_{0}$ : when the magnitude of the potential barriers is small $T_{c}$ is approximately $T_{0}$, then it falls as $\tilde{u}_{0}$ increases, and for sufficiently high potential barriers the critical temperature is proportional to $\tilde{u}_{0}^{-1 / 2}$. In addition, for $(a+b) / \lambda_{0}<1$ we clearly see that

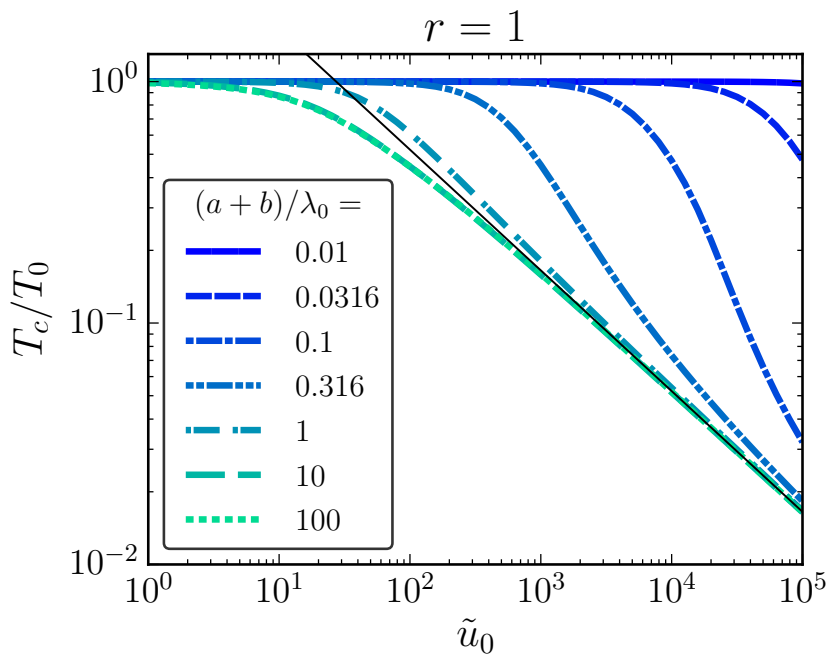

Fig. 1: (Color online) $T_{c} / T_{0}$ as a function of $\tilde{u}_{0}$. The solid, thin line indicates the behavior given by (6). 
$T_{c}$ is very close to $T_{0}$, even for very high potential barriers. This behavior is due to the fact that, when the potential period becomes very small with respect to $\lambda_{0}$, the energy spectrum of the bosons approaches more and more to that of a free particle, so $T_{c}$ tends to $T_{0}$. It seems as if the thin potential wells are unable to capture bosons and all of them have energies above $V_{0}$ behaving as a free IBG.

In the other hand, we found that when $\left[(a+b) / \lambda_{0}\right] r \sqrt{\tilde{u}_{0}} /(1+r) \gg 1$, the energy bands whose energies are less than $\tilde{u}_{0}$ are very narrow, and they resemble the energy levels of a particle in a box of width $a$. In this case we can approximate the energies of the first band through a Taylor series up to second order derivatives of $\varepsilon_{z}\left(k_{z}\right)$ around $k_{z}=0$,

$$
\varepsilon_{z} \approx \varepsilon_{0}+\left.\frac{1}{2} k_{z}^{2} D_{k_{z}}^{2} \varepsilon_{z}\right|_{k_{z}=0} .
$$

The linear term on $k_{z}$ is zero because $\varepsilon_{z}(0)=\varepsilon_{0}$ is a minimum of the energy. Using this approximation, and taking the condition that $(a+b) / \lambda_{0} \gg 1$, the behavior of $T_{c} / T_{0}$ is given by the relation

$$
\frac{r \tilde{u}_{0}^{1 / 2}}{(1+r) \zeta(3 / 2)} \frac{T_{c}}{T_{0}}+\frac{1}{1+r}\left(\frac{T_{c}}{T_{0}}\right)^{3 / 2}-1=0,
$$

which is potential period independent, therefore $T_{c} / T_{0}$ depends only on $\tilde{u}_{0}$ and $r$. When $r \sqrt{\tilde{u}_{0}} /(1+r) \gg 1$ the second term of $(5)$ becomes negligible, and we obtain the concrete dependence of the critical temperature shown in Fig. 1 as a solid thin line,

$$
\frac{T_{c}}{T_{0}}=\frac{1+r}{r} \zeta(3 / 2) \tilde{u}_{0}^{-1 / 2} .
$$

Fig. 2: (Color online) $T_{c} / T_{0}$ as a function of the potential period.

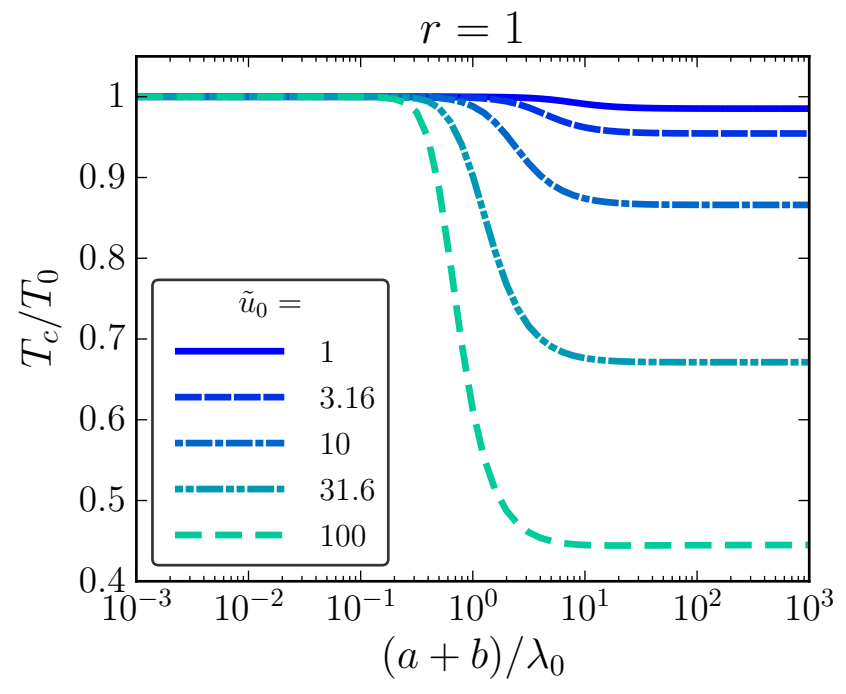


The numerical results plotted in Fig. 1 1 supports that Eq. (6) is an excellent limit relation when $\tilde{u}_{0}$ is very large in comparison with $\left[(a+b) / \lambda_{0}\right]^{-2}$, even when $(a+b) / \lambda_{0}$ is smaller than unity. The behavior of $T_{c}$ as a function of the potential period can be seen in Fig. 2. where we note that $T_{c}$ approximates to $T_{0}$ when the potential period is small, then it falls as the period increases, and finally it goes to a certain value that is $\tilde{u}_{0}$ and $r$ dependent, but becomes $(a+b) / \lambda_{0}$ independent. This constant value is given by solving (5).

It is interesting to see how the critical temperature does not necessarily approaches to $T_{0}$ again as the potential period keeps increasing, unlike the case of a Dirac comb potential 7 . Even when the empty space between barriers becomes larger (as well as the barriers width), the distribution of the bosons over the bands is very different to that of the free Bose gas. The dependence on $\tilde{u}_{0}$ and $r$ that appears on the first term of (5) comes from the distribution of the bosons over the first energy band. The dependence on $r$ in the second term of (5) comes from the boson distribution over the rest of the bands. Only in the case when $r=0$ the critical temperature is $T_{0}$. This is expected because $r=0$ means no barriers, i.e., $b=0$, or $a \rightarrow \infty$ while $b$ remains constant. In both cases it is clear why we recover the behavior of the free Bose gas.

\section{Dimensional crossover and energy spectrum}

We have calculated the specific heat at constant volume for several combinations of the parameters (see Fig. 4 of Ref. [8]). The specific heat has a peak due to the Bose-Einstein condensation of the confined IBG at a critical temperature $T_{c} \leq T_{0}$. Above $T_{c}$ the specific heat has a complex structure, and for some combinations of the parameters of the system it shows a dimensional crossover within an interval of temperatures where it falls to a minimum approximately equal to unity; in this interval the system behaves

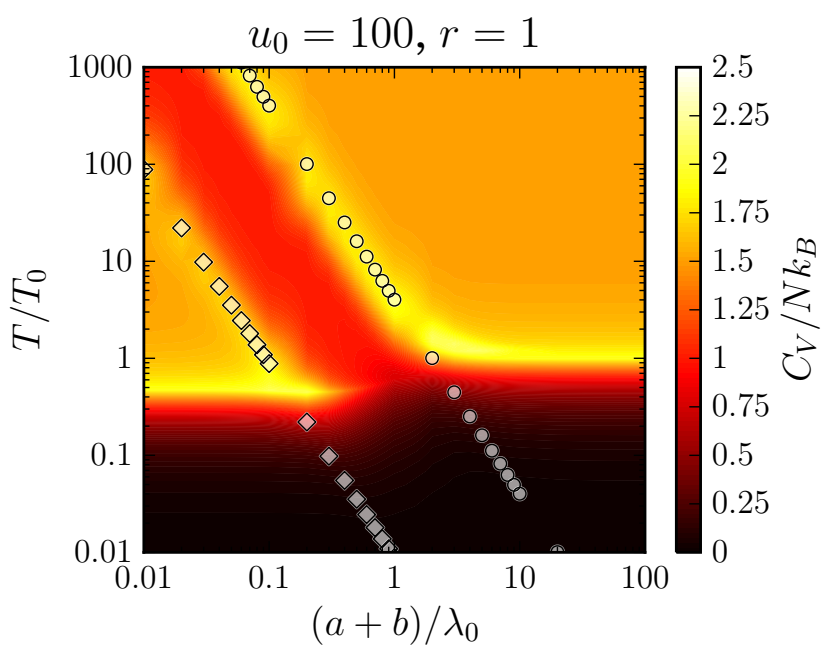

Fig. 3: (Color online) $C_{V} / N k_{B}$ contours as a function of the potential period and the temperature. 
as a $2 \mathrm{D}$ gas, trapped by the potential in one direction but remaining free in the other two. The minimum, which is a consequence of the trapping effects imposed by the potential barriers could become a plateau when the potential magnitude is increased. In addition to the minimum, the specific heat has two maxima. The maximum at the lower temperature marks the crossover from the $3 \mathrm{D}$ behavior of the gas to a $2 \mathrm{D}$ behavior. The maximum at higher temperature represents the return of the system to its three-dimensional behavior or it may be seen as the superior limit of the temperature interval where the crossover occurs.

In order to give a explanation about the meaning and position of the specific heat maxima in terms of the energy spectrum characteristics, we realized a contour plot of $C_{V} / N k_{B}$ as a function of $(a+b) / \lambda_{0}$ and $T / T_{0}$, for a system with $u_{0}=100$ and $r=1$ (see Fig. 3 ) where the maximum positions are highlighted with bright yellow.

When we associate the temperature of the maxima $T_{\max }$ with a yet unknown energy chunk $\Delta \varepsilon_{z}$ characteristic of the energy spectrum such that $\Delta \varepsilon_{z}=k_{B} T_{\max }$, we found that both lower and higher maxima temperatures follow the relation

$$
\frac{T_{\max }}{T_{0}}=\frac{1}{4 \pi} \Delta \bar{\varepsilon}_{z}\left(\frac{a+b}{\lambda_{0}}\right)^{-2}
$$

where $\Delta \bar{\varepsilon}_{z}$, the energy chunk given in recoil energy unit divided by $\pi^{2}$, has a different value depending on whether we are describing the maximum at lower or higher temperature.

The maximum that occurs at the higher temperature is reproduced using an energy $\Delta \bar{\varepsilon}_{z}$ given by the difference between the bottom edge of the first energy band and the bottom edge of the second energy band. In the Fig. 3 this is shown with white circles. The maximum at the lower temperature occurs when $\Delta \bar{\varepsilon}_{z}$ is approximately equal to $1 / 4$ of the width of the first energy band. In the Fig. 3 this relation is shown with white diamonds.

Fig. 4: $\quad($ Color online) $C_{V} / N k_{B}$ contours as a function of the potential period and the temperature.

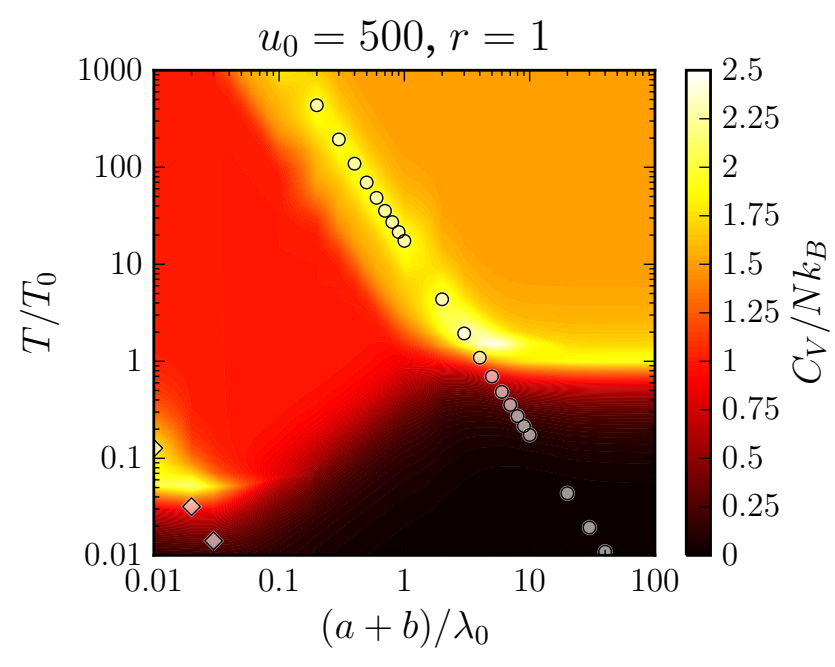


As a more general result, it is found that as the magnitude of $u_{0}$ increases the energy chunk needed to reproduce the $T_{\max }$ of the maximum at higher temperatures, eventually corresponds to the energy difference between the bottom edges of the first and the third, fourth or subsequent bands of the confined Bose gas energy spectrum. An example of this behavior is show in Fig. 4 for the system with $u_{0}=500$ and $r=1$, where the energy chunk $\Delta \bar{\varepsilon}_{z}$ associated to the maximum at the higher temperature corresponds to the difference between the bottom edges of the third and first band. However, the dependence of the energy chunk on $u_{0}$ and $r$ is not trivial to find, and currently we have no analytical expression to determine it. It is noticeable that, to reproduce the maximum at lower temperature with expression (7) we always use $\Delta \bar{\varepsilon}_{z}$ approximately to $1 / 4$ of the width of the first energy band of the spectrum, i.e., in this case $\Delta \bar{\varepsilon}_{z}$ value is potential strength independent.

\section{Conclusions}

In summary, we analyzed the behavior of the BEC critical temperature of an IBG confined by an infinite stack of slabs as well as the effects of the external periodic potential on the dimensional crossover, the specific heat maximum positions and their relation with the distribution of allowed and forbidden bands in the energy spectrum. We found that the critical temperature $T_{c}$ decreases continuously as the potential barrier height increases, and it becomes proportional to $\tilde{u}_{0}^{-1 / 2}$ when $\tilde{u}_{0}$ is much larger than $\left[(a+b) / \lambda_{0}\right]^{-2}$. It is found that this behavior is universal as it does not depends on the potential period, the barrier width nor the barrier separation. In addition, we found that as the period increases the $T_{c}$ becomes a constant value given by Eq. (5) which is spatial period independent. On the other hand, the specific heat at constant volume shows a dimensional crossover within a certain interval of temperatures where it behaves like a $2 \mathrm{D}$ gas due to the trapping by the potential barriers. The region where the dimensional crossover occurs as well as the specific heat maximum positions depend directly on the forbidden and allowed bands distribution in the energy spectrum. We were able to find a relation between the maximum positions and characteristic energies of the energy spectrum. Although the specific heat maximum position, at higher temperature, is reproduced using the Eq. (7) with $\Delta \bar{\varepsilon}_{z}$ the energy difference between the bottom edges of the second and first bands, or, depending on the potential strength, the difference between the bottom edges of the third and first, the bottom edges of the fourth and first and so on, we highlight that the specific heat maximum position at the lower temperature is reproduced using the Eq. (7) with $\Delta \bar{\varepsilon}_{z}$ being potential strength independent and equal to $1 / 4$ of the width of the first band.

Acknowledgements. We acknowledge partial support from grants PAPIITDGAPA-UNAM IN-105011 and IN-111613, CONACyT 221030 and PAEP UNAM. 


\section{References}

1. J. G. Bednorz and K. A. Müller, Z. Phys. B Condensed Matter 64, 189 (1986); M. R. Norman and C. Pépin, Rep. Prog. Phys. 66, 1547 (2003).

2. M. Greiner, O. Mandel, T. Esslinger, T. Hänsch, W. Theodor, and I. Bloch, Nature 415, 39 (2002).

3. H. J. Lauter, H. Godfrin, V. L. P. Frank, P. Leiderer, Phys. Rev. Lett. 68 2484 (1992); L. M. Steele, C. J. Yeager, D. Finotello, Phys. Rev. Lett. 71, 3673 (1993); K. Shirahama, K. Yamamoto, Y. Shibayama, J. Phys. Soc. Jpn. 77, 111011 (2008)

4. G. Bastard, Phys. Rev. B 24, 5693 (1981); G. Bastard, Phys. Rev. B 25, 7584 (1982); Semiconductor Superlattices: Growth and Electronic Properties edited by Holger T. Grahn (World Scientific Publishing, USA, 1995).

5. F. Ancilotto, M. M. Calbi, S. M. Gatica, M. W. Cole, Phys. Rev. B 70, 165422 (2004); B. Marcone, E. Orlandini, F. Toigo, F. Ancilotto, Phys. Rev. B 74, 085415 (2006).

6. R.L. de Kronig and W.G. Penney, Proc. Lond. Ser. Math. Phys. Sci. 130, 499 (1930).

7. P. Salas, F. J. Sevilla, M. Fortes, M. de Llano, A. Camacho, and M. A. Solís, Phys. Rev. A 82, 033632 (2010); P. Salas et al., (2015), "Specific heat of underdoped cuprates as a function of doping", submitted for publication to JLTP.

8. O. A. Rodríguez and M. A. Solís, Journal of Low Temp. Phys. 175, 435, (2014). 